\title{
Diagnostic yield of deep biopsy via endoscopic submucosal dissection for the diagnosis of upper gastrointestinal subepithelial tumors: a systematic review and meta-analysis
}

\author{
Amaninder Dhaliwala, Sindhura Kollib, Banreet Singh Dhindsac, Harmeet Singh Mashiana ${ }^{a}$, \\ Neil Bhogala, Ishfaq Bhat ${ }^{a}$, Shailender Singh ${ }^{a}$, Douglas G. Adler ${ }^{d}$
}

University of Nebraska Medical Center, Omaha; The Brooklyn Hospital Center, New York; University of Nevada Las Vegas School of Medicine; University of Utah School of Medicine, Huntsman Cancer Center, Salt Lake City, Utah, USA

\section{Abstract}

\begin{abstract}
Background Conventionally, endoscopic ultrasound-guided fine-needle aspiration and biopsy (EUS-FNA)/EUS-FNB) has been used for tissue diagnosis of upper gastrointestinal (GI) subepithelial tumors (SETs). However, deep biopsy (DB) via endoscopic submucosal dissection (ESD) is emerging as an alternative technique, given the inadequate tissue sampling with EUSFNA/EUS-FNB. Our aim was to conduct a systematic review and meta-analysis to report the overall diagnostic yield of DB via ESD for upper GI SETs.
\end{abstract}

Methods PubMed, Cochrane Library and Web of Science databases were searched to identify studies (from commencement to Oct 2017) that reported the DB via ESD technique for diagnosis of upper GI SETs. The primary outcome of interest was the method's overall diagnostic yield and the secondary outcome was to the occurrence of complications. The meta-analysis was performed using the DerSimonian and Laird random-effects model.

Results A total of 7 studies, comprising 209 patients with a mean age of 57.3 years, were included in the final meta-analysis. The overall pooled diagnostic yield of DB via ESD for upper GI SETs was $95 \%$ (95\% confidence interval [CI] 84.91-99.98, $I^{2}=78.2 \%$ ). Overall, pooled outcomes of major bleeding and perforation in our meta-analysis was noted in $0.07 \%\left(95 \% \mathrm{CI} 0.00-2.32, I^{2}=0 \%\right)$ and $0 \%$ (95\% CI $\left.0.00-1.70, I^{2}=0 \%\right)$ respectively. Data regarding major bleeding and perforation rates were not reported in 2 studies. Substantial heterogeneity was observed in our meta-analysis.

Conclusion DB via ESD is an effective and safe procedure for diagnosing upper GI SETs. Further multicenter randomized controlled trials are needed to validate these findings.

Keywords Deep biopsy, submucosal dissection, subepithelial tumors

Ann Gastroenterol 2020; 33 (1): 1-8

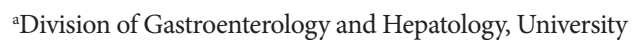
of Nebraska Medical Center, Omaha (Amaninder Dhaliwal, Singh Mashiana, Neil Bhogal, Ishfaq Bhat, Shailender Singh); ${ }^{\text {Department of }}$ Internal Medicine, The Brooklyn Hospital Center, New York (Sindhura Kolli); ' Department of Internal Medicine, University of Nevada Las Vegas School of Medicine (Banreet Singh Dhindsa); ${ }^{\mathrm{d} D e p a r t m e n t ~ o f ~}$ Gastroenterology and Hepatology, University of Utah School of Medicine, Huntsman Cancer Center, Salt Lake City, Utah (Douglas G. Adler), USA

\section{Conflict of Interest: None}

Correspondence to: Douglas G. Adler, MD, FACG, AGAF, FASGE, Professor of Medicine, Director of Therapeutic Endoscopy, Director, GI Fellowship Program, Gastroenterology and Hepatology, University of Utah School of Medicine, Huntsman Cancer Center, 30 N 1900 E, Room 4R118, Salt Lake City, Utah 84132, USA, e-mail: douglas.adler@hsc.utah.edu

Received 16 August 2019; accepted 14 October 2019; published online 29 November 2019

DOI: https://doi.org/10.20524/aog.2019.0444

\section{Introduction}

Subepithelial tumors (SETs) within the uppergastrointestinal (GI) tract, which comprises the esophagus, stomach and duodenum, arise from the mucosa layer under the epithelium without changes typically being visible on the mucosa. SETs make up less than $1 \%$ of all GI tumors and have varying rates of malignant transformation [1,2]. They are divided into nonneoplastic lesions (varices), neoplastic lesions with minimal malignant potential (lipomas, leiomyomas, pancreatic rests), and neoplastic lesions with a higher malignant potential (GI stromal tumors [1], carcinoid tumors, schwannomas) [1,3]. GISTs, the most common type of SET, have a malignancy potential of $30 \%$, making an accurate diagnosis essential for directing treatment [4].

When an upper GI SET is encountered during an endoscopy, the current standard of care is an endoscopic 
ultrasound (EUS) examination, usually accompanied by an EUS-guided fine-needle aspiration and/or biopsy (EUS-FNA)/ (EUS-FNB) to obtain tissue for cytologic/histologic analysis and immunohistochemical (IHC) testing.

IHC testing possesses the 2-pronged utility of confirming the diagnosis of GIST and differentiating it from other hypoechoic SETs of varying malignancy potential, such as carcinoid tumors or schwannomas [5,6]. However, the quality of the biopsy is a major determinant of the diagnostic yield, which in turn dictates the type of treatment, surveillance and expected rate of recurrence, while deterring unnecessary interventions based on a low-yield biopsy [7].

EUS-FNA is the standard, given its diagnostic accuracy of $100 \%$ in SETs larger than $40 \mathrm{~mm}$. In SETs between 20 and $40 \mathrm{~mm}$ its accuracy diminishes to $86-91 \%$, and in SETs smaller than $20 \mathrm{~mm}$ it becomes $50-70 \%$ [8]. The addition of a forward-viewing and curved linear-array echoendoscope (FVCLA-ES) improves accuracy to $81 \%$ [8], while the use of EUS-FNB improves the overall accuracy rate to $87 \%$ in small lesions [9].

An emerging alternative to the current standard is a deep biopsy (DB) via endoscopic submucosal dissection (ESD), which has demonstrated a high diagnostic yield at about $95 \%$, even with lesions smaller than $20 \mathrm{~mm}[5,7,10,11]$. It is generally an outpatient procedure that begins with epinephrine in saline being injected into the submucosal layer of the visible lesion. A flex knife makes a 5-mm hole through which a larger IT-2 knife is inserted. Approximately, $15 \mathrm{~mm}$ of the mucosa and submucosa are dissected. Multiple biopsies are taken and site is closed with clips [7]. Factors such as the average time dedicated to the procedure by experienced endoscopists (51 $\mathrm{min}$ on average for ESD vs. $18 \mathrm{~min}$ on average for EUS-FNA), outpatient feasibility for EUS-FNA versus hospitalization and monitoring required post-ESD, and complications pertaining to each procedure, are crucial details when comparing the 2 efficacious methods [12,13]. This systematic review and meta-analysis aimed to analyze the overall diagnostic yield and complications of the DB via ESD technique.

\section{Materials and methods}

The objectives, primary and secondary outcomes, search strategy, inclusion and exclusion criteria, and methods for study selection, data extraction and data synthesis in this meta-analysis were defined in a protocol in advance as per guidelines [14].

\section{Information sources and search strategy}

A literature search was performed within the databases of PubMed, Cochrane Library and Web of Science. Various amalgamations of the following keywords were utilized: "submucosal dissection", "intraepithelial gastric lesions", "deep biopsy”, "endoscopic submucosal dissection", “GISTs", "stromal tumors", "upper gastrointestinal subepithelial tumors", "submucosal lesion", "EUS” and "EGD".

\section{Inclusion and exclusion criteria}

Studies that examined the diagnostic yield of deep biopsies via ESD of upper GI SETs were included. Study designs included comprised case series, prospective and retrospective studies. Case reports, editorials and studies reported only in abstract form were all excluded. Included studies were written in English and published within 10 years retrospectively from August 2017. Studies with incomplete data and those not meeting the inclusion criteria were excluded.

\section{Data extraction and quality assessment}

Data was extracted and verified by independent reviewers. The initial screening stage consisted of searching for studies in which the title and abstract delineated clear examination of the diagnostic yield of DB via ESD in upper GI SETs in human subjects. In the subsequent stage, a complete examination of the article was undertaken to ensure relevance to our points of interest. Data collected included the first author, year of publication, years the study spanned, type of study, total number of patients, size and type of GI SETs, and diagnostic yield of DB via ESD. We assessed the quality of included studies using a scale modified from the Newcastle-Ottawa scale for cohort studies [14].

\section{Statistical analysis}

Our primary outcome of interest was the overall diagnostic yield of DB via ESD for upper GI SETs as compared to the current standard of EUS with FNA/FNB. Diagnostic yield is defined as the positive predictive value of the preoperative diagnosis as compared to the postoperative pathological diagnosis of tissue obtained by DB via ESD. Our secondary outcome was to study adverse events, including but not limited to perforation and clinically significant bleeding. The meta-analysis was performed using the DerSimonian and Laird random-effects model. Measuring the binary outcomes of improved diagnostic yield of DB via ESD was achieved by measuring the pooled risk difference. All pooled rates were calculated with a $95 \%$ confidence interval (CI) and with a respective P-value, considered statistically significant when $<0.05$. A forest plot was constructed for the pooled estimates of the primary outcome of diagnostic yield of DB via ESD, as well as the secondary outcomes. Variation of results across studies due to heterogeneity rather than chance was expressed by $I^{2}$. An $I^{2}$ value $>50 \%$ suggests significant heterogeneity. 


\section{Results}

\section{Literature search}

Using our search criteria, a total of 183 citations were identified. After duplicate studies were removed, 122 citations remained to be reviewed. On further application of the inclusion and exclusion criteria, 7 studies that discussed the use of deep biopsy via ESD in the diagnosis of upper GI SETs were included. The meta-analyses included 7 independent cohort studies totaling 209 patients (Fig. 1). All of them were single-center based and all procedures were performed by 1-2 experienced advanced endoscopists at each center. Five of the studies had 18 or more patients, while 2 studies contained 8 and 9 respectively. Design-wise, one was retrospective, one was a case series, and the remaining 5 were prospective. All of them were full publications and in original manuscript form. Of the 209 patients, 94 (44.9\%) were male and 115 (55.1\%) were female, and their mean age was 57.3 years (range: $22-88$ years) [5,7,13,15-18]. Details of these studies are shown in Table 1.

\section{Overall diagnostic yield}

In our study population, the overall pooled diagnostic yield of DB via ESD for upper GI SETs was 95\% (95\%CI

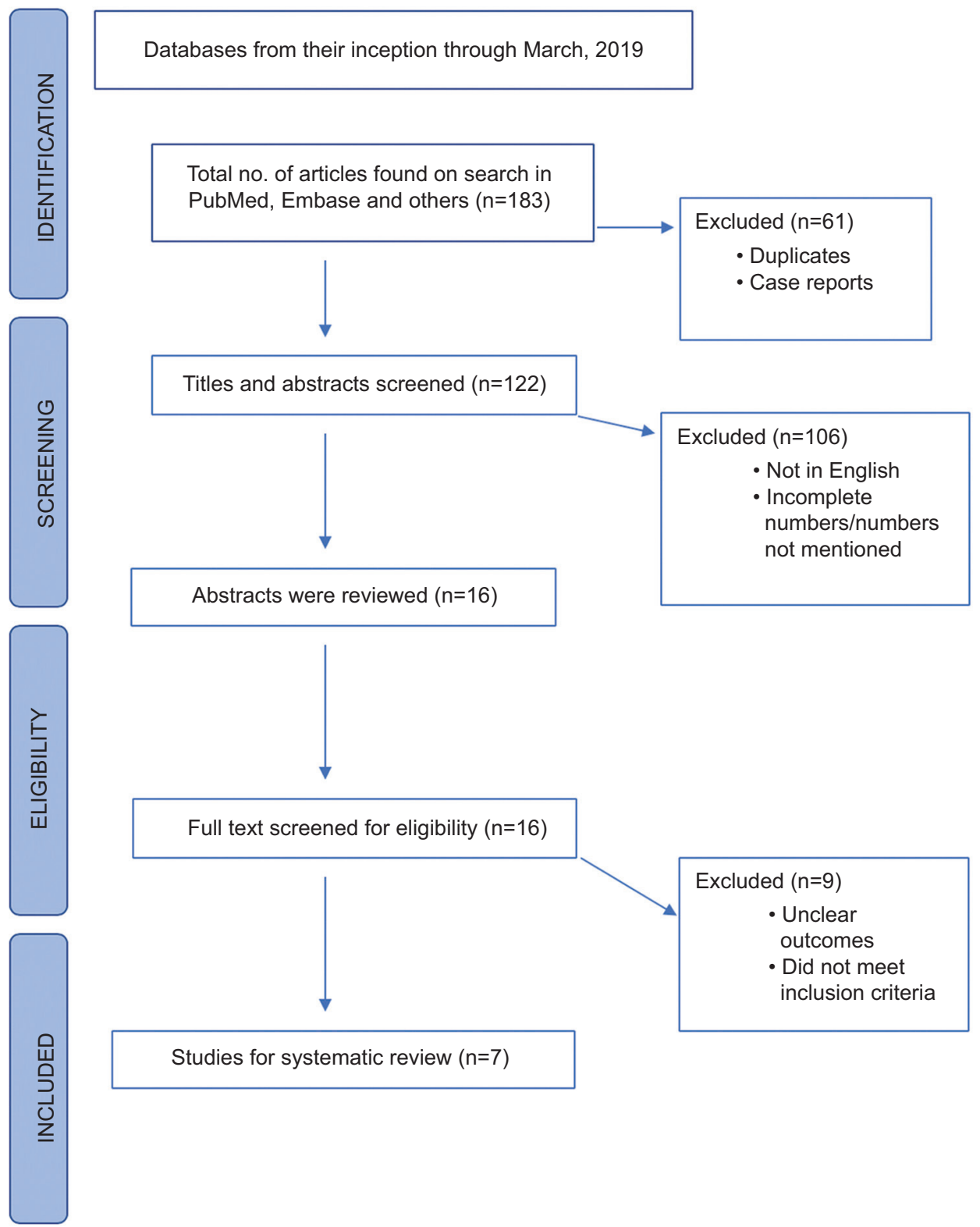

Figure 1 Study selection process in accordance with preferred reporting items for systematic reviews and meta-analysis (PRISM) statement 
84.91-99.98, $I^{2}=78.2 \%$ ) as outlined in the forest plot (Fig. 2). Of them, GISTs were diagnosed in $37.48 \%$ (95\%CI 22.25-53.97, $\left.I^{2}=80.17 \%\right)$, followed by leiomyomas $(29.19 \%$, 95\%CI 22.92 $\left.35.85, I^{2}=0.00 \%\right)$, ectopic pancreas $(13.67 \%, 95 \% \mathrm{CI} 6.97-21.84$, $\left.I^{2}=49.65 \%\right)$, lipomas $\left(3.05 \%\right.$, 95\%CI $\left.0.68-6.48, I^{2}=0.00 \%\right)$, and others $\left(3.98 \%, 95 \%\right.$ CI $\left.0.75-8.75, I^{2}=33.49 \%\right)$. "Others" encompassed entities such as fibromas, duplication cysts, or Brunner's gland hyperplasia.

The mean tumor size was $18.8 \mathrm{~mm}$ (95\%CI 16.3-21.4, $\left.I^{2}=97 \%\right)$. A majority of the upper GI SETs were located in the stomach $\left(96 \%, 95 \%\right.$ CI $\left.83.77-100, I^{2}=84.6 \%\right)$, followed by the esophagus $\left(2.18 \%, 95 \%\right.$ CI $\left.0.00-10.20, I^{2}=77.34 \%\right)$ and

Table 1 Details of the studies included in our meta-analysis

\begin{tabular}{|c|c|c|c|c|c|}
\hline Reference & Year of publication & Country & Study type & Years & No. of patients \\
\hline Kobara et al [13] & 2017 & Japan & Prospective & 2011-2016 & 43 \\
\hline Vaicekauskas et al [5] & 2016 & Lithuania & Case Series & 2012-2014 & 38 \\
\hline Jung et al [16] & 2016 & Korea & Prospective & 2013-2014 & 42 \\
\hline Tae et al [7] & 2014 & Korea & Prospective & 2010-2013 & 40 \\
\hline Kobara et al [18] & 2013 & Japan & Prospective & 2011-2012 & 8 \\
\hline Lee et al [17] & 2011 & Korea & Prospective & 2010 & 9 \\
\hline
\end{tabular}

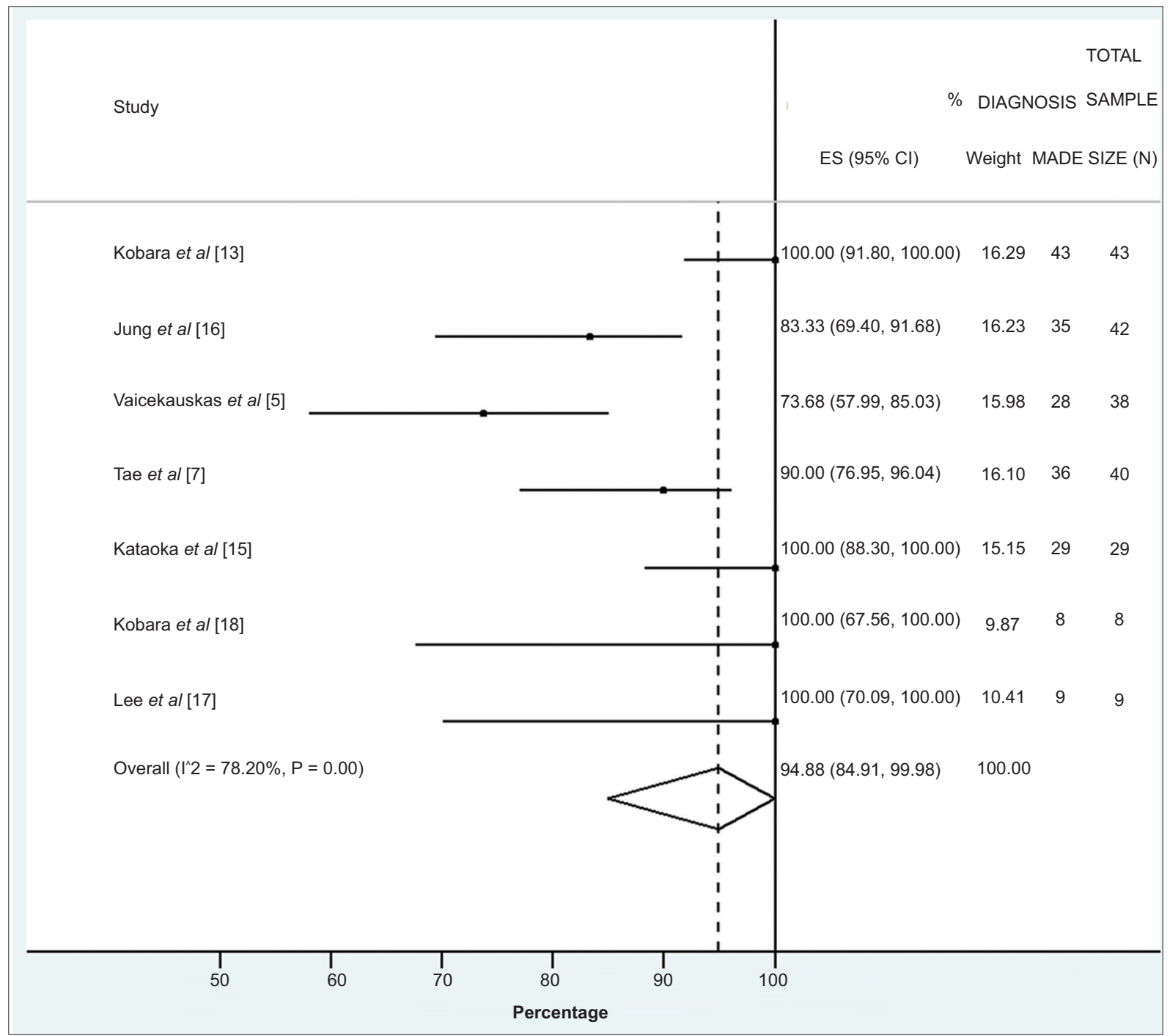

Figure 2 Forest plot depicting overall diagnostic yield of deep biopsy via endoscopic submucosal dissection 
duodenum (1.09\%, 95\%CI 0.00-4.67, $\left.I^{2}=36.02 \%\right)$, while none were detected by the gastroesophageal junction within our population $(n=209)$.

\section{Adverse events}

Data regarding major bleeding and perforation rates were studied and reported in 6 studies, while 2 omitted any mention of them. At this stage, the authors conducted a subgroup analysis of the studies that did report adverse events. Overall, the pooled rate of major bleeding was $0.07 \%$ (95\%CI 0.00-2.32, $\left.I^{2}=0 \%\right)$, while the perforation rate was $0 \%$ (95\%CI $0.00-1.70$, $\left.I^{2}=0 \%\right)$. Figs. 3 and 4 are forest plots depicting major bleeding and perforation, respectively.

\section{Validation of meta-analysis results}

\section{Heterogeneity}

Based on Q statistics, and $I^{2}$ analysis, substantial heterogeneity was observed for the overall pooled diagnostic yield of DB via ESD for upper GI SETs (78.2\%). Within the category of upper GI SETs, the heterogeneity was noted in the diagnostic yield of GISTs $\left(I^{2}=80.17 \%\right)$. No heterogeneity was noted in the adverse events: major bleeding and perforation rates.

\section{Publications bias and quality of the studies}

The power to detect publication bias was low, given the small number of studies for comparison. The quality of the studies was assessed by the Newcastle-Ottawa scale (Table 2), which showed that all studies were of high quality with regard to representation of inclusion cohorts and study outcomes.

\section{Discussion}

We performed a systematic review of studies that examined $\mathrm{DB}$ via ESD in the diagnosis of GI SETs and found that they demonstrated a high overall diagnostic yield. A subset analysis

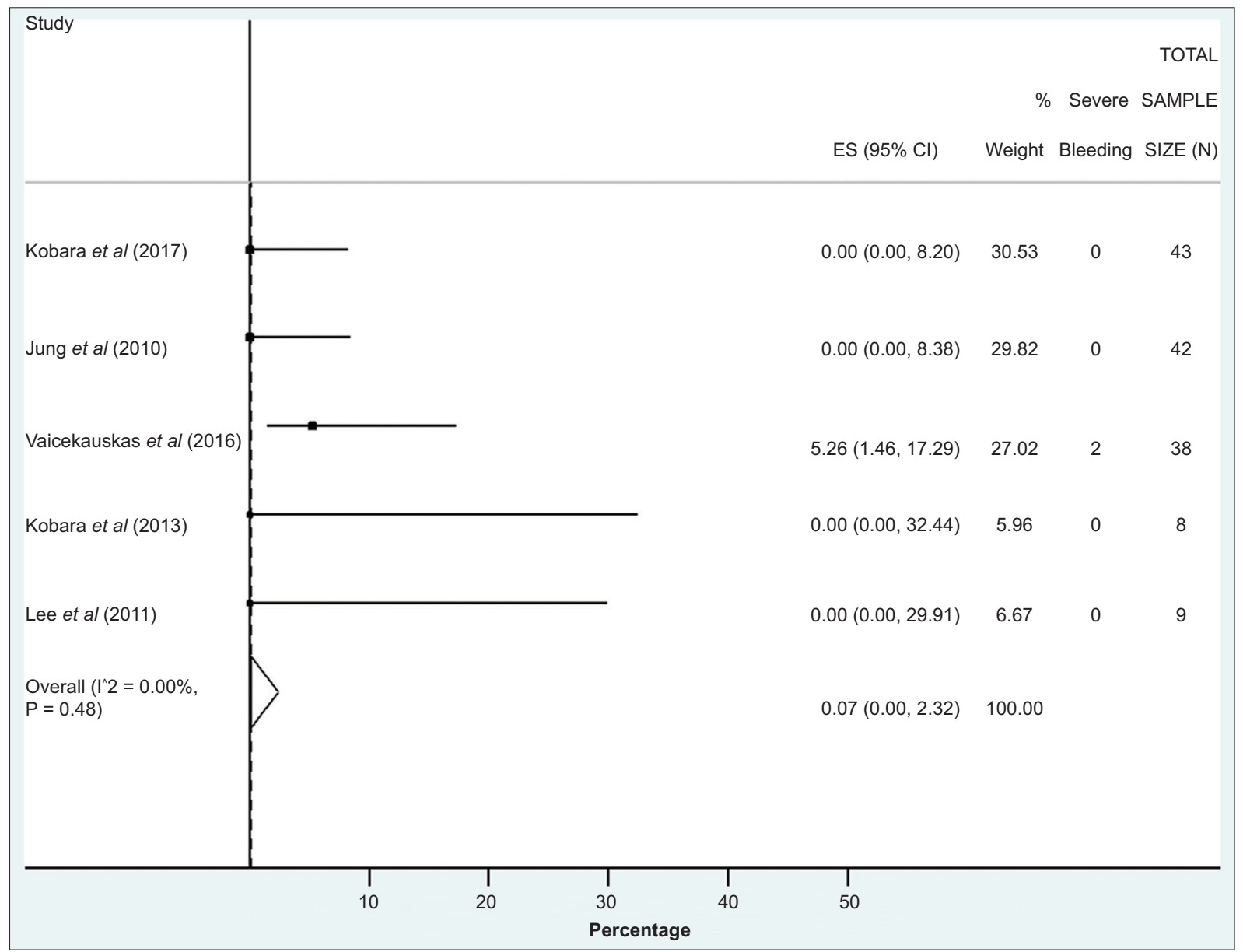

Figure 3 Forest plot analysis of bleeding per procedure 


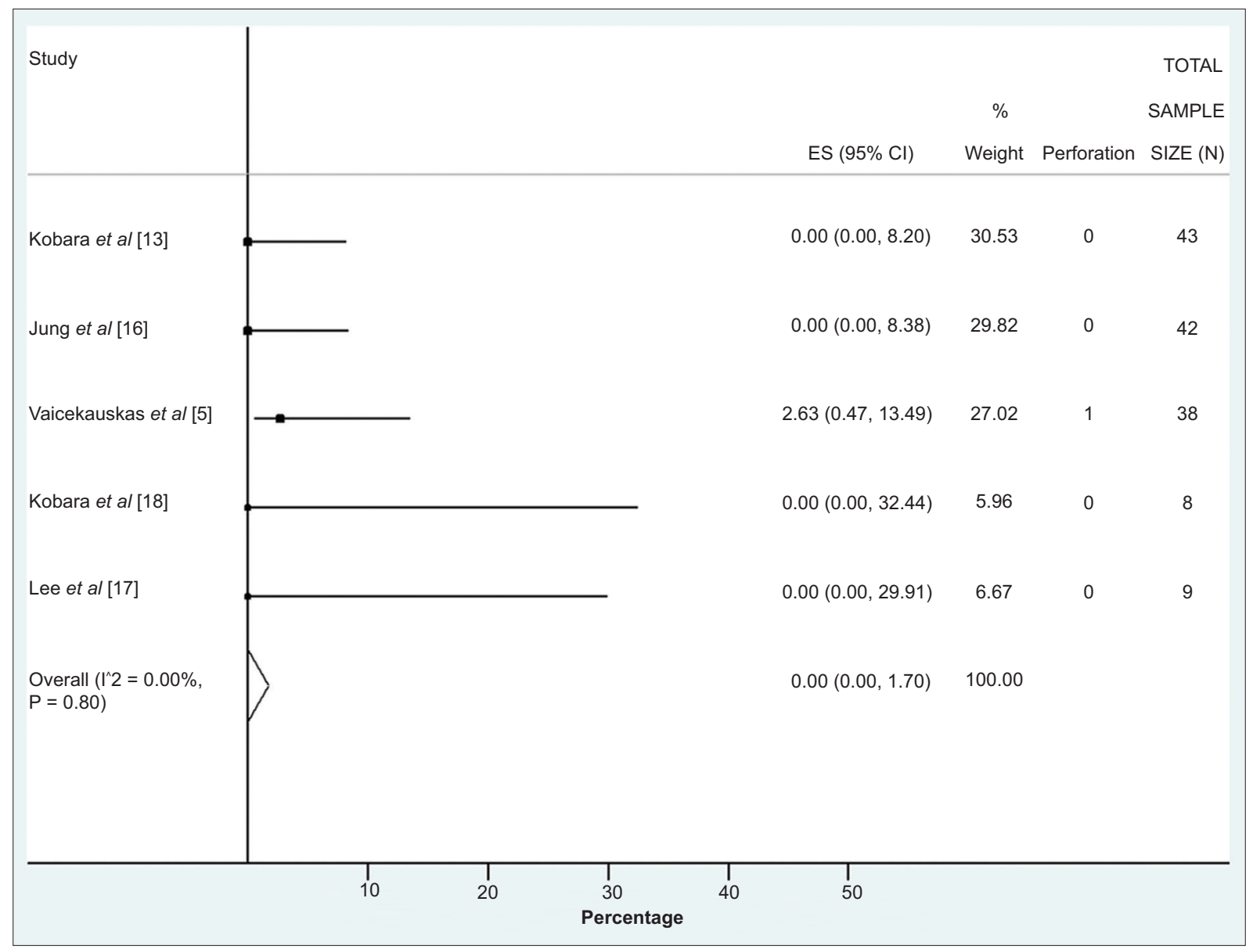

Figure 4 Forest plot analysis of perforations per procedure

of the adverse events related to DB via ESD showed minimal and infrequent complications, indicating that the technique is a safe alternative to EUS-FNA.

Current guidelines dictate that GISETs $20 \mathrm{~mm}$ require resection, in view of their high probability of malignancy, while GI SETs $<20 \mathrm{~mm}$ are considered benign and EUS surveillance or resection is recommended $[5,7,13,15,19]$. Factors with high malignancy potential are GI SETs located in the upper or middle portions of the stomach, or when they originate from the muscularis propria level $[13,15]$. Given the possibility of a malignant GIST, many endoscopists currently obtain a biopsy of GI SETs less than $20 \mathrm{~mm}$ for cytologic/histologic analysis and IHC testing for confirmation. This allows one to diagnose and differentiate between the various subtypes of GI SETs, which range from GISTs (which have a malignancy rate up to $30 \%$ ) to benign lipomas, in order to guide treatment. For this reason, the type of biopsy that will return the highest diagnostic yield is preferred. DB via ESD shows a higher diagnostic yield than the current standard of EUS-FNA, especially when targeting smaller lesions.

EUS-FNA's diagnostic accuracy is largely dependent on the size of the lesion. It ranges from $100 \%$ in lesions larger than $40 \mathrm{~mm}$ to as low as $50 \%$ in lesions smaller than $20 \mathrm{~mm}$ [8]. EUSFNB has an overall accuracy of $87 \%$. EUS-FNA's limitations are largely logistical: difficulty in maneuvering the echoendoscope in a small number of locations, including the proximal gastric cardia or certain portions of the third duodenum, and, rarely, the inability to adequately sample a lesion once it has been identified [13]. This can result in tissue samples inadequate for IHC staining for markers such as CD34, S-100, CD117, $\mathrm{Ki}-67$ or desmin, or for calculating an accurate mitotic index to assess malignant potential [7]. These limitations are more pronounced in GI SETs less than $20 \mathrm{~mm}$, a subgroup with a very low risk of malignancy [7].

Higher diagnostic yields with DB via ESD may occur because the size of the tissue sample is sufficient for IHC testing, even with smaller lesions. Additional benefits with GI SETs, such as the shorter procedure time (10 vs. an average of $39 \mathrm{~min}$ for EUS-FNA), prevention of repeat procedures or unnecessary surgical exploratory laparotomy, and earlier initiation of a treatment plan based on biopsy results, make DB via ESD a favorable approach $[5,7,15,16,19]$.

In our meta-analysis, DB via ESD had an overall diagnostic yield of $95 \%$, even with lesions smaller than $20 \mathrm{~mm}$. It provided adequately sized tissue samples to determine the mitotic activity with $>5$ mitoses $/ 50$ high power field diagnosing malignant activity and a higher rate of recurrence, as well as for IHC staining. Tae et al pointed out that the biopsies from EUS-FNA had a mitotic disparity compared to the tissue from 
Table 2 Newcastle-Ottawa scale for study quality assessment

\begin{tabular}{|c|c|c|c|c|c|c|}
\hline \multirow[t]{2}{*}{ Author } & \multirow[t]{2}{*}{ Study type } & \multirow[t]{2}{*}{ Cohort/Case-control } & \multirow[t]{2}{*}{ Year } & \multicolumn{3}{|c|}{ Newcastle-Ottawa scale } \\
\hline & & & & Selection & Comparability & Outcomes \\
\hline Kobara et al [13] & Prospective & Cohort & 2017 & $* * *$ & * & $* *$ \\
\hline Vaicekauskas et al [5] & Case Series & Cohort & 2016 & $* * *$ & * & ** \\
\hline Jung et al [16] & Prospective & Cohort & 2016 & $* * * *$ & $* *$ & $* * *$ \\
\hline Tae et al [7] & Prospective & Cohort & 2014 & $* * * *$ & $* *$ & $* * *$ \\
\hline Kataoka et al [15] & Retrospective & Cohort & 2013 & $* * *$ & $* *$ & $* * *$ \\
\hline Kobara et al [18] & Prospective & Cohort & 2013 & $* * *$ & * & $* *$ \\
\hline Lee et al [17] & Prospective & Cohort & 2011 & $* * *$ & * & $* *$ \\
\hline
\end{tabular}

the surgically resected SETs, indicating gratuitous resections in $46 \%$ of the SETs [7]. Jung et al point out that, given the heterogeneous distribution of mitotic activity, biopsies should be obtained from the area with the most mitotic activity, possible with DB via ESD [16]. A prospective study of 87 patients by Tae et al demonstrated that none of the patients in the DB via ESD group had unnecessary surgery, while in the EUS-FNA group, $42.9 \%$ of the patients had unneeded surgical procedures as the resected tissue was ultimately judged to be benign [7].

DB via ESD also provides greater flexibility when approaching GI SETs located in the gastric fundus and duodenum [13]. This is especially important, as Kataoka et al described a higher malignancy potential in GI SETs located in the upper and middle divisions of the stomach [15].

In our meta-analysis of $\mathrm{DB}$ via ESD, major bleeding was noted in only 2 patients, while only 1 patient had perforation, managed endoscopically. Two studies did not document any adverse events. Successful and rapid endoscopic hemostasis without subsequent sequelae was achieved in cases of bleeding [17]. In the remaining studies within our metaanalysis, complications were infrequent, minimal, and without associated morbidity or mortality. Concurrently, the possibility of seeding is also minimal, as the dissection takes place within the submucosa or muscularis layer.

Some limitations were present in our meta-analysis. All of our studies were observational, heterogeneous small studies and no randomized controlled trials were included. They were all single-center based observational studies. Two studies did not describe the adverse events related to $\mathrm{DB}$ via ESD. A majority of the procedures, if not all, were performed by experienced advanced endoscopists, so it is unclear to what extent that influenced both the diagnostic accuracy and the adverse complications.

In conclusion, DB via ESD is an effective and safe procedure for diagnosing upper GI SETs, with an overall diagnostic yield of $95 \%$ and minimal adverse events. Its benefits, including accuracy in diagnosis with GI SETs smaller in size $(<20 \mathrm{~mm})$ or located in anatomically challenging sites, a potentially shorter procedure time, prevention of costly and time-consuming surgical exploration, paired with minimal and easily controlled complications, make it a viable alternative to EUS sampling in the approach to upper GI SETs. Multicenter randomized control trials are needed to further validate these findings in comparison to EUS sampling approaches.

\section{Summary Box}

\section{What is already known:}

- Conventionally, endoscopic ultrasound (EUS) guided fine-needle aspiration and biopsy (EUSFNA/EUS-FNB) has been used for tissue diagnosis of upper gastrointestinal (GI) subepithelial tumors (SETs)

- EUS-FNA is the standard, given its diagnostic accuracy of $100 \%$ in lesions larger than $40 \mathrm{~mm}$

- In lesions, between $20 \mathrm{~mm}$ and $40 \mathrm{~mm}$ its accuracy diminishes to $86-91 \%$, and in lesions less than $20 \mathrm{~mm}$ it becomes $50-70 \%$

\section{What the new findings are:}

- Deep biopsy (DB) via endoscopic submucosal dissection (ESD) is emerging as an alternative technique for the tissue diagnosis of upper GI SETs, given the inadequate tissue sampling with EUS-FNA/EUS-FNB for lesions smaller than $20 \mathrm{~mm}$

- DB has a high diagnostic yield at about $95 \%$, even with lesions less than $20 \mathrm{~mm}$, for tissue diagnosis of upper GI SETs

- The procedure has a few adverse events, with minimal postprocedural bleeding and perforation rates

\section{References}

1. Hwang JH, Rulyak SD, Kimmey MB; American Gastroenterological Association Institute. American Gastroenterological Association Institute technical review on the management of gastric subepithelial masses. Gastroenterology 2006;130:2217-2228.

2. Zhao X, Yue C. Gastrointestinal stromal tumor. J Gastrointest Oncol 
2012;3:189-208.

3. Franco MC, Schulz RT, Maluf-Filho F. Opinion: How to manage subepithelial lesions of the upper gastrointestinal tract? World $J$ Gastrointest Endosc 2015;7:1262-1267.

4. Søreide K, Sandvik OM, Søreide JA, Giljaca V, Jureckova A, Bulusu VR. Global epidemiology of gastrointestinal stromal tumours (GIST): a systematic review of population-based cohort studies. Cancer Epidemiol 2016;40:39-46.

5. Vaicekauskas R, Stanaitis J, Valantinas J. Efficacy of deep biopsy for subepithelial lesions in the upper gastrointestinal tract. Wideochir Inne Tech Maloinwazyjne 2016;11:192-199.

6. Yamabe A, Irisawa A, Bhutani MS, et al. Usefulness of endoscopic ultrasound-guided fine-needle aspiration with a forward-viewing and curved linear-array echoendoscope for small gastrointestinal subepithelial lesions. Endosc Int Open 2015;3:E161-E164.

7. Tae HJ, Lee HL, Lee KN, et al. Deep biopsy via endoscopic submucosal dissection in upper gastrointestinal subepithelial tumors: a prospective study. Endoscopy 2014;46:845-850.

8. Attila T, Aydin. Lesion size determines diagnostic yield of EUS-FNA with onsite cytopathologic evaluation for upper gastrointestinal subepithelial lesions. Turk J Gastroenterol 2018;29:436-441.

9. Iwai $\mathrm{T}$, Kida $\mathrm{M}$, Imaizumi $\mathrm{H}$, et al. Randomized crossover trial comparing EUS-guided fine-needle aspiration with EUS-guided fine-needle biopsy for gastric subepithelial tumors. Diagn Cytopathol 2018;46:228-233.

10. Shimamura Y, Hwang J, Cirocco M, May GR, Mosko J, Teshima CW. Efficacy of single-incision needle-knife biopsy for sampling subepithelial lesions. Endosc Int Open 2017;5:E5-E10.

11. Suzuki T, Arai M, Matsumura T, et al. Factors associated with inadequate tissue yield in EUS-FNA for gastric SMT. ISRN
Gastroenterol 2011;2011:619128.

12. Liu F, Zhang S, Ren W, et al. The fourth space surgery: endoscopic subserosal dissection for upper gastrointestinal subepithelial tumors originating from the muscularis propria layer. Surg Endosc 2018;32:2575-2582.

13. Kobara H, Mori H, Nishimoto N, et al. Comparison of submucosal tunneling biopsy versus EUS-guided FNA for gastric subepithelial lesions: a prospective study with crossover design. Endosc Int Open 2017;5:E695-E705.

14. Stroup DF, Berlin JA, Morton SC, et al. Meta-analysis of observational studies in epidemiology: a proposal for reporting. Meta-analysis Of Observational Studies in Epidemiology (MOOSE) group. JAMA 2000;283:2008-2012.

15. Kataoka M, Kawai T, Ikemiyagi $\mathrm{H}$, et al. Clinicopathological characteristic and clinical handling of the patients with $2 \mathrm{~cm}$ or less gastric GISTs. Springerplus 2013;2:469.

16. Jung YS, Lee H, Kim K, Sohn JH, Kim HJ, Park JH. Using forceps biopsy after small submucosal dissection in the diagnosis of gastric subepithelial tumors. J Korean Med Sci 2016;31:1768-1774.

17. Lee HL, Kwon OW, Lee KN, et al. Endoscopic histologic diagnosis of gastric GI submucosal tumors via the endoscopic submucosal dissection technique. Gastrointest Endosc 2011;74:693-695.

18. Kobara H, Mori H, Fujihara S, et al. Bloc biopsy by using submucosal endoscopy with a mucosal flap method for gastric subepithelial tumor tissue sampling (with video). Gastrointest Endosc 2013;77:141-145.

19. Marcella C, Shi RH, Sarwar S. Clinical overview of GIST and its latest management by endoscopic resection in upper GI: a literature review. Gastroenterol Res Pract 2018;2018:6864256. 